capabilities and scientific techniques, another factor of 1,000 is not out of the question."

\section{ALL THE SMALL THINGS}

Nanoscale connectomes have been completed in two species: the nematode Caenorhabditis elegans ${ }^{1}$, in 1986, and the larva of a marine organism known as Ciona intestinalis ${ }^{2}$, in 2016.

These neural maps are a powerful winnowing tool. "There are a lot of hypotheses that have been refuted by the $C$. elegans wiring diagram," says Bobby Kasthuri, a neuroscientist at Argonne National Laboratory in Lemont, Illinois. If an observation about the worm's nervous system or behaviour can be easily explained by the wiring diagram, there's no need for further experiments; researchers can move on to more fruitful lines of inquiry. But when the connectome doesn't offer a ready explanation for results, it can indicate productive directions of research for scientists to explore.

Some researchers do question the focus on nanoscale connectomes. The enormous amount of time, effort and money that goes into such projects might be overkill, says Anthony Movshon, a neuroscientist at New York University in New York City who studies the visual system. When it comes to complex brains such as those of mice or humans, "I don't need to know the precise details of the wiring of each cell and each synapse in each of those brains," Movshon says. "What I need to know, instead, is the organizational principles that wire them together." This, he suggests, can be gleaned with a coarser level of resolution.

Yet the nanoscale connectome is a goal that captures the imaginations of many scientists. They say that this work could help to unravel the origins of mental-health conditions and lead to more informed treatments, as well as have applications in a host of fields, including artificial intelligence (see page S15) and energy-efficient computation.

\section{PROJECT PROLIFERATION}

To map the nanoscale connectome of C. elegans, in the 1980s, researchers led by biologist Sydney Brenner at the University of Cambridge, UK, thinly sliced the millimetrelong worms and photographed each slice using a film camera mounted to an electron microscope. In the resulting images, they then painstakingly traced by hand the pathways of neurons and the connections between them.

But C. elegans has a mere 302 neurons and around 7,600 synapses. The methods used to produce its connectome simply weren't practical to use in larger nervous systems. Researchers did not think seriously about embarking on considerably larger projects until 2004, when physicist Winfried Denk and neuroanatomist Heinz Horstmann, then both at the Max Planck Institute for Medical Research in Heidelberg, Germany, proposed using an automated microscope to slice and image the brain and software to stack and align the resulting images ${ }^{3}$.

One of the largest complete nanoscale

connectomes to be released represents a roughly 100-micrometre cube of mouse retina containing around 1,000 neurons and 250,000 synapses. Denk, now director of the Max Planck Institute of Neurobiology in Martinsried, Germany, and his collaborator Moritz Helmstaedter, co-director of the Max Planck Institute for Brain Research in Frankfurt, Germany, published the analysis ${ }^{4}$ in 2013. But the mouse-brain cubic-millimetre project will be looking at 100,000 neurons, and other, similar programmes are also under way.

"A cubic millimetre is a size that seems to be sufficient, at least for the neurons in the centre of that grain of sand, to get most of their local connections," says Nuno da Costa, a neuroscientist at the Allen Institute. The mouse-brain project will therefore enable scientists to explore complete local circuits, rather than single neurons with a sparse network of connections. The work being conducted at the Allen Institute is part of a collaboration with researchers at Baylor College of Medicine in Houston, Texas, Princeton University in New Jersey and Harvard University in Cambridge, Massachusetts, known as Machine Intelligence from Cortical Networks, which is funded by the US government.

Its progress has led some to predict that the nanoscale connectome of a complete mouse brain - likely to produce around one exabyte (one billion gigabytes) of data - could be mapped in the next decade. "It would require many laboratories," says Jeff Lichtman, a neuroscientist at Harvard University. "But it's doable," he says, "and that's exciting."

Others remain cautious. "There are so many logistic challenges" to a project of that size, says Stephen Plaza, a computer scientist at the Howard Hughes

Medical Institute

Janelia Research

Campus in Ashburn,

Virginia. He thinks

that the field should

"We're still in the trainingwheels stage of connectomics."

target intermediary-

scale projects before tackling something as complex as the mouse brain. "We're still in the training-wheels stage of connectomics," he says.

Plaza manages one such project. Called FlyEM, it aims to produce a connectome of the central nervous system of the fruit fly Drosophila melanogaster. His team expects to release data on roughly one-third of the D. melanogaster brain in early 2020 . Plaza expects that the connectome of the entire central nervous system - composed of about 100,000 neurons and 100 million connections in the fly's brain alone, plus a similar number of neurons and synapses in the ventral nerve cord (roughly equivalent to the spinal cord of vertebrates) - will follow a few years later.

Meanwhile, Lichtman is working on the zebrafish (Danio rerio) connectome, as well as analysing a small piece of the human brain - a sample of the medial temporal gyrus obtained from a person who was undergoing brain surgery for epilepsy. That piece is also roughly one cubic millimetre in volume, but to capture the full thickness of the human cortex, the sample is shaped like a slab, rather than a cube.

Denk and his colleagues are mapping portions of the connectome in the zebra finch (Taeniopygia guttata), a small bird whose process of song learning can yield insights into human speech. And Kasthuri has a number of projects in progress. "Now that there is a lot of data available on the circuitry of the mouse brain, I think the best way to do it is to either look across species or look across development," he says. "The best information will come from comparing that diagram to other things."

To that end, Kasthuri aims to map the visual part of the brain in non-human primates, as well as in an octopus (Octopus bimaculoides). "It's probably the creature that is the most alien to us that's still smart," he says, of the octopus. "So, I'm interested in comparing the wiring of that brain to the wiring of the mouse brain."

Kasthuri is also working on the full connectomes of young mice and octopuses; comparing these immature connectomes to those of adult animals could offer insights into how the brain learns from experience. Owing to its small size, he hopes to map the youngoctopus connectome in about one year.

\section{AI SPY}

Now that the researchers at the Allen Institute have finished imaging their cubic millimetre of mouse brain, they have passed on the data to Sebastian Seung, a neuroscientist and computer scientist at Princeton University. Seung's lab will align the resulting images, and then annotate the synapses and trace, or segment, the estimated four kilometres of nerve fibre that are contained within the volume.

Segmentation has long been the rate-limiting step in connectomics. It can take weeks to trace by hand the path of a single neuron through a stack of electron micrographs. But now, artificial intelligence is getting involved. Seung's team has developed a machine-learning algorithm that can evaluate images pixel by pixel to determine the location of neurons.

Computers can perform segmentation faster than the human eye, which cuts down the time it takes to trace neurons to a matter of minutes or hours. But they aren't as accurate: algorithms can miss out bits of neuron or incorrectly merge two neurons into one. People are therefore still needed to check the reconstruction. Seung is tackling this requirement through crowdsourcing and, specifically, an online game called Eyewire, in which players are challenged to correct mistakes in the rough draft of a connectome. Launched in 2012, Eyewire has 290,000 registered users who have collectively put in an effort that is equivalent to 32 people working full time for 7 years, says Amy Robinson Sterling, executive director of Eyewire.

So far, players have been tracing cells in the mouse retina. They've contributed to the discovery of six types of neuron, which players chose to name after ancient Greek deities. 
Sterling and her team are preparing a new version of the game, called Neo, that will be used with the mouse visual-cortex data set.

Neo's interface is based on Neuroglancer, a program developed by Google that visualizes flat, black-and-white electron micrographs as a colourful 3D forest of neurons. Many nanoscale connectome-mapping efforts use the program to visualize data.

Google has also developed an algorithm for neuron segmentation. A team led by Viren Jain at Google AI, in Mountain View, California, has designed a machine-learning algorithm called a flood-filling network, which builds structures from a point in an image, rather than trying to define the boundaries of all neurons at once. "It's a little bit like the way a human would colour in a colouring book," Jain says. His team is applying the technique to FlyEM data and has constructed a roughdraft connectome of a whole fly brain that was imaged by another team at Janelia Research Campus. They are also working with data from the labs of Denk and Lichtman.

"It's truly beautiful to look at," Lichtman says of the result, noting that the algorithm is able to trace neurons faster than his team can collect imaging data. "We're not able to keep up with them," he adds. "That's a great place to be."

Jain strikes a more cautious note, and points out that as scientists take on ever larger projects, segmentation algorithms have to become more accurate to keep feasible the amount of human checking that is required.

\section{CLOSE LOOK}

Meanwhile, scientists are honing microscopy techniques to produce sharper, more-detailed images at a much quicker pace, in anticipation of taking on the nanoscale connectomes of large, mammalian brains.

The conventional approach to microscopy in connectomics is a type of electron microscopy known as serial-section electron microscopy. Researchers embed neural tissue in plastic, and cut it into slices that are a fraction of the thickness of a human hair. They then mount the slices on a specialized tape and feed the result - which looks remarkably similar to film on a reel - through the microscope.

The advantage of this method is that the sample is preserved and can be re-imaged, if needed. But no matter how precisely it is done, cutting the sample inevitably results in distortions that make it difficult to align the images.

A newer approach, known as focused ion beam scanning electron microscopy (FIBSEM), uses a beam of charged ions to shave away a thin layer of a tissue sample. The microscope captures an image of the freshly exposed surface, and then the process is repeated. The FlyEM sample represents the first substantial volume to be imaged by this method.
Although it lacks speed, one advantage of FIB-SEM is that the resolution of the images produced is the same in all three dimensions, rather than being coarser along the vertical axis. However, samples can be imaged only once, because they are vaporized in the process. In addition, the field of view is very small, which makes it difficult to apply to larger samples. (Even the fruit-fly brain, which is roughly the size of a poppy seed, has to be chopped into smaller chunks.) A method called gas cluster ion beam scanning electron microscopy (GCIB-SEM), developed by Kenneth Hayworth, a neuroscientist at Janelia Research Campus, works similarly but has a larger field of view, which makes it more feasible for use in imaging larger brains.

GCIB-SEM might also be more compatible with multibeam electron microscopes, which researchers hope will speed up image acquisition. Numerous electron beams scan a sample at the same time, which enables the micro-

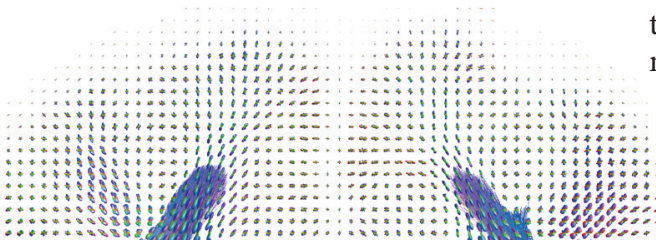

producing data, another challenge is looming: how to make sense of it all. "We have so much processed data at our fingertips," Reid says. "A large number of scientists could routinely discover new things on this data set. Many more than we could possibly hire."

There is also the issue of linking nanoscaleconnectome data with that produced by other large-scale neuroscience projects such as the Human Connectome Project. That effort used magnetic resonance imaging to scan the brains of about 1,200 people to define millimetrewide tracts of nerve fibres that connect regions of the brain. The result was a map known as the macroconnectome.

"The biggest problem in neuroscience is the problem of scale," says David Edwards, a neonatologist at Kings College London. He is part of the Developing Human Connectome Project, which is wrapping up its effort to scan the brains of hundreds of fetuses in the womb, as well as those of both full-term and premature babies. "There are great things being done at the macroscale, great things being done at the microscale, great things being done at population level," Edwards says. "But there are very few ways of linking those together."

Fresh sources of data that are, in some ways, even more detailed than the nanoscale connectome, are also emerging. For example, the connectome only provides information about the location of synapses, not their molecular composition. "I see that as a gap that needs to be bridged," says Seth Grant, a molecular neuroscientist at the University of Edinburgh, UK. "If you can't bridge it, you don't find your way to the genome." And those genomic insights, Grant suggests, will be essential for working out how evolution and genetics govern brain function.

Enter the synaptome. In a 2018 paper, Grant and his team catalogued one billion synapses across the whole mouse brain ${ }^{5}$, which enabled them to define 37 subtypes on the basis of protein content, size and shape, and to identify patterns of subtypes that characterize various brain regions. The team has also begun to match the subtypes with the connections that they make. "Marrying up the synaptome with the connectome," Grant says, "is going to be one of the next frontiers."

scope to capture hundreds of millions of pixels per second. Lichtman is using a machine produced by Carl Zeiss that has 61 beams, and Denk has one with 91 beams. And electron microscopes with hundreds of beams are on the way, which might eventually capture a gigapixel of imaging data every second.

\section{MAKE IT MEANINGFUL}

But speed creates its own problems. Now that nanoscale connectome projects are rapidly
Sarah DeWeerdt is a science writer based in Seattle, Washington.

1. White, J. G., Southgate, E., Thomson, J. N. \& Brenner, S. Philos. Trans. R. Soc. Lond. B 314, 1-340 (1986).

2. Ryan, K., Lu, Z. \& Meinertzhagen, I. A. eLife $\mathbf{5}$ e16962 (2016)

3. Denk. W. \& Horstmann, H. PLoS Biol. 2, e329 (2004).

4. Helmstaedter, M. et al. Nature 500, 168-174 (2013).

5. Zhu, F. et al. Neuron 99, 781-799 (2018) 